\title{
Destiny or Free Will Decision? A Life Overview from the Perspective of an Informational Modeling of Consciousness Part I: Information, Consciousness and Life Cycle
}

\author{
Florin Gaiseanu* \\ Science and Technology of Information, Romania \\ *Corresponding author: Florin Gaiseanu, Science and Technology of Information, Romania
}

Submission: 眥 October 01, 2018; Published: 眥 January 03, 2019

\begin{abstract}
We drive our lives permanently by decisions YES/NO, and even we no longer distinguish the elementary intermediary steps of such decisions most often, they form stereotyped chains that once triggered, they run unconsciously, daily facilitating our activities. We lead our lives actually by conscious decisions, each of such decisions establishing our future trajectory. The YES/NO dipole is actually the elemental evaluation and decisional unit in the informational transmission/reception equipment and lines and in computers, respectively. Based on a binary probabilistic system, this is defined as a unit of information (Bit). We operate therefore as an informational system and we actually live in a bipolar universe, which is fundamentally informational. Indeed, the laws of nature and its equilibrium or steady state conditions are based on bipolar units with opposite characteristics, such as action/reaction, attraction/rejection, gravity/anti-gravity, matter/antimatter, entropy/anti-entropy, to enumerate just a few examples. As part of this bipolar universe, we are also bipolar entities connected to information and matter. Starting from the informational features of the human being, seven informational components are identified, forming the informational system of the human body, distinguished by their different functions, reflected at the conscious level through the center Iknow (the memory, including whole life experience), Iwant (decisions center), Iove (emotions), Iam (body status), Icreate (informational genetic transmitter), Icreated (genetic generator inherited from parents) and Ibelieve, which is the gateway to the antientropic component, favorable to maintain the life structure and functioning. Taking into account the characteristics of these centers, it is discussed the life cycle and are deduced suitable conclusions concerning an optimal, active lifestyle, that would contribute to a successful life, aging and destiny.
\end{abstract}

\section{Introduction}

When we talk about destiny, we usually think in a supernatural force that decides for us, and which we cannot oppose to, whatever we would do or think. There may be forces in the world, which we really cannot resist to. But the destiny is particularly connected to our trajectory in life, related also to the way in which we think and act. That's why the destiny is everyone's, individually related to each of us and to our own life.

Have we often contemplated on how the destiny is capable to influence us? Do we know enough about our decisional system and on the way, we have the possibility to lead our lives? This paper aims to analyze the life cycle from the perspective of an informational modeling of consciousness that reveals and put forward the informational structure of the human body, helping us to know ourselves and to behave accordingly with the circumstances. Consciousness has been and has remained an extremely important issue from this point of view, although it is still necessary to discover many of its secrets. Particularly, if we are only referring to the tools for the investigation of consciousness, it is remarkable that the science and technology have been able to allow the using of non-invasive and non-destructive methods in the brain activity observation, based on electrical and magnetic effects. These investigations have permitted remarkable applications, helping the immobilized people to command their assisting electro-mobile systems [1], by the use of direct or wireless thought transducers.

But these results are not enough to explain more complex phenomena, such as those associated with the near-death experiences [2,3], or the remote communication by thoughts [4], so it was necessary to approach consciousness taking into account more phenomena, related for instance to the quantum behavior of the particles and microstructures [5], and more recently to information as a specific component of matter, playing a fundamental role in explaining both the normal and extra-normal properties of mind $[6,7]$. Indeed, this approach allowed not only the understanding of 
these properties, but also an assessment of an extremely delicate theme, that of the existence of consciousness after death. In the Part I of this work we present the main informational components of consciousness, which we define as cognitive centers, allowing an overview of life and its evolution cycle from such a perspective. The definition and understanding of their functions allow observations and conclusions on how we can live our life optimally, accordingly to its laws and not outside of them, contributing in such manner through our decisions to a harmonious collaboration with the environment and with the society where we live in. Specifically, these conclusion are referred in the Part I especially to an optimal lifestyle for the maintenance of the mental and cognitive efficiency, while in the Part II for the follow of an equilibrated lifestyle by a personal control, necessary to assure a successful life, aging and destiny.

\section{YES/NO alternatives as an informational decisional unit}

Every moment of life we decide. This affirmation could seem to be exaggerated, but if we carefully analyze each of the activities that we perform daily to achieve a certain purpose, we will notice that this statement is true. Many of our behavior sequences are no longer aware, because they have become automated mechanisms. But we learned them in a past time and became automatic by a systematic repetition process. Our body is an adaptable system, always ready to learn, and probably this was and remains a key of the human evolution. New situations require a decision, always a choice between YES and NO, even if the path to the final objective requires a chain no matter as long of YES/NO elementary decisional steps. A decision we take every moment can mark the life and the trajectory that we will follow in the future. Everyday activity is actually composed by a sum of elementary YES/NO decisions, which traces our way in the life and directs us to a goal, although some of them have become undetectable at conscious level. A large part of the decisions is addressed to the body itself, in order to achieve its movements, while others act as decision elements inside of the mental operational system. But YES/NO binary alternative expresses nothing else that the information unit (Bit), as it is known for example from the computer operations. The computers work with numbers in the binary system, 0 and 1 , actually functioning as switches, so 1 Bit is expressed in terms of probability in such binary systems. In the information science and technology, information is defined as a measure of the certainty, while entropy express the contrary, namely the quantity of uncertainty, or disorder. We therefore observe that we operate as intelligent, informational systems, able to capture external information by means of our senses (seeing, hearing, smelling, taste, touching) and inner information (thirst, hunger, pain and various impulses), to analyze it and to develop a decision or chain of decisions. This decision is passed to the execution organs, mainly the muscles, or is stored in the acquisition and storage system, which is the memory. We are therefore some informational systems, prepared and able to collect, process and transmit information to the inside and/or the outside of the body [6].

But we are not only the product of the environment, but also the instrument of its transformation, which can intervene on it and modify it. We are therefore dynamic reactive systems: we capture information and react by decision, changing not only the environment by direct action on it, but also our own way of thinking and thus our behavior, depending on the modification of our criteria system [7,8]. We actually live in a bipolar world, although at a first glance we are not accustomed to think in that way. But yet it seems so. It is enough to note that any "Good" has its associated "Bad", the Good / Bad unit is constituted as a bipolar unit of two contraries, opposing each other. Indeed, we have not only relevant examples from physics, such as action/reaction, order/ disorder, entropy/anti-entropy, gravity/anti-gravity, matter/ antimatter $[9,10]$, but also from everyday life we can easily discover them we ourselves. Many qualities or characteristics of an object or entity of any kind has its opposite. We therefore live in a world of opposite features, so in an informational world, operating as a YES/NO binary system. We accept by YES what we consider to be in accordance with our personal value scale, and classify by NOT the opposite one, rejecting it. It depends on us any choice YES or NO under some given circumstances, we are therefore our own decision-maker, the drivers of our life by our own free-will. The YES/NO unit actually ensures by its essence the balance in nature. It has recently been shown that the gravity is actually an entropic force [11], and the time arrow in an antimatter system (assimilated with dark matter $[9,10]$, is oriented in the opposite direction to the time arrow known in the matter system [12], characteristics which we are not accustomed to operate with, but which succeed to explain the specific phenomena associated to the near-death experiences and "abnormal" or "para"-normal properties of mind such as premonition and psychokinesis [6,7]. Therefore, we have to know that even the time is bipolar, and it can be manifested in two opposite directions. Does would exist a connection between the bipolar universe we live in, and these physical properties? Do they influence or even determine our lives? Of course, YES, because we are part of this system and in correlation with it. Does nature seem to seek a balance between the bipolar contrary phenomena? YES, it looks like. And alive alike? It seems that YES, as we will explain further.

\section{The informational system of the human body and consciousness as a result of its activity}

The informational system of the human body according to the informational modeling $[3,6,7]$ is represented in Figure 1. This figure reveals first of all the bipolar character of this system, connected to matter and to information. The system of components marked by white color represents the conscious level of consciousness, which we define as the Operative Information System (OIS), oriented to immediate adaptation to the environment. Its components can be defined and described as follows. (i) The Center of Acquisition and Storing of Information (CASI), dedicated to the capture and storing of information, consisting in external and internal sensors for information capture plus memory, where information is stored; this is projected into consciousness as a cognition center Iknow (which we refer for simplicity as to Ik), which actually includes all of our life experience. (ii) The Center of Decision and Command (CDC) is the operative center that takes over the information 
from CASI and can analyze it, generating the decision and the corresponding command to the execution elements (EE), especially the muscles; it is projected into consciousness as the center Iwant (Iw), representing the own will, so the personal position (Attitude) in relation to a certain analyzed situation. (iii) The Info-Emotional
System (IES), reflected in consciousness by the center Ilove (II), includes all the sensations, emotions, feelings and emotional states of the body, treated as reactions of the organism to the captured or analyzed information.

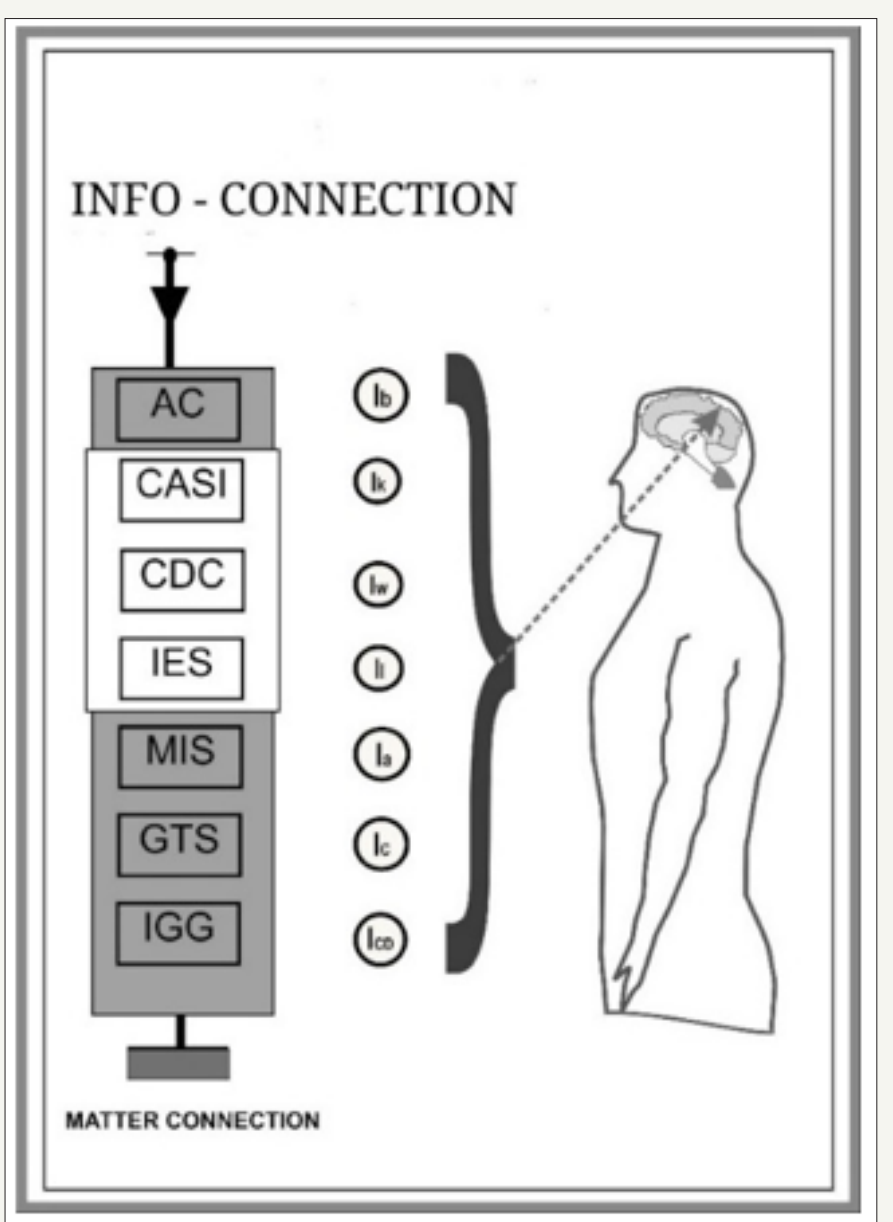

Figure 1: Schematic representation of the informational system of the human body with its components and the projected informational (cognitive) centers in consciousness.

The Programmed Information System (PIS) consists in components that are mainly dedicated to the processing and (re) structuring of matter (marked in Figure 1 in gray color) and can be assimilated to the subconscious level. The components of this system (noted as a continuation of the previously started numbering), can be defined as follows. (iv) The Maintenance Information System (MIS), which mainly manages automatically the acquisition, processing and distribution of organic and inorganic matter, i.e. the foods (nourishment, water, air) necessary to support the body, defined as Informed Matter (IM); the projection of this system in consciousness is given by the center Iam (Ia), expressing mainly the needs, status of the body and self-consciousness. (v) The Genetic Transmission System (GTS) ensures the generation process of genetically codified matter and its transmission to the next generation, as the genetic information output, in order to preserve the survival of the species; this system is projected in consciousness as the center Icreate (Ic), reflecting the associativity, responsibility behavior and productivity within the family and the society. (vi) The Info-Genetic Generator (IGG), reflected in consciousness by the center Icreated (Icd), under the form of predispositions, talents, mentality, represents the input genetic gate, including the specific genetic information gathered from parents. (vii) Anti-entropic Connection (AC) is the connection gate with the anti-entropic force of the universe, which ensures the structural and functional balance of living beings in opposition to the entropic (destructive) force; AC is projected into consciousness by the center Ibelieve (Ib), felt as a source of trust, stability and health.

As it can be observed from the above presentation, the bipolar structure of the universe described in this modeling seems to coincide with the Taoist vision of the universe, based on the Yin/ Yang dipole, while the seven-centers architecture of the human informational system described here with the seven-chakras structure described and propagated by some oriental traditional cultures [13]. 


\section{Life evolution from the perspective of the informational modeling of consciousness}

We are born as organisms from a dual combination of informed matter from parents, with a certain finite amount of codified information. The separation into two sexes, not only at the human beings, but also in most of the living creatures, is a reflection of the bipolar character of the universe, as it will be shown in detail in a volume that will be published in the future. The genetic codified information follows the body development program through IGG. Within the maternal body, the formation of the future child seems to take place following an informational matrix, specifically structured, determining the shape and functions of each organ, and the general body architecture. An anti-entropic force seems to be involved in the drawing/structuring of the future child body, according to the human prototype contained in this origin genetic matrix, the egg. During the first years of life, when the nervous system is under a process of intensive development, the neurons multiply with an incredible speed, often replacing the previous ones to form new connections [14], so the received information from the family environment will have a defining character. The learning effort to walk and to talk is colossal, but the information system of the body is suitable and capable of this effort. The rules and the distinction between Good and Bad are consolidated into the informational structure, found out under a full formation process, becoming decision criteria most often for the entire life. The Ik center develops itself accordingly with the quantity of the received and processed information. We no longer distinguish the progressive steps YES/NO which we have learned for to stand up and go for instance, these steps have become automatic informational chains. The execution of such chains has been acquired and integrated into the subconscious informational system.

The repetition is the fundamental procedure which such an integration takes place through. We are therefore adaptable systems, able to acquire new features by learning and by a repetition process. The learning is an associative mechanism, because a new piece of information is added to a similar one, already stored in CASI. The operating system (Iw) during this period takes particularly into account the impulses coming from the internal sensors of hunger, thirst, but also from external ones, stimulating the imitation desire. Self-consciousness (Ia) develops more slowly, the general development of consciousness being dominated during the first years of life by the centers Iw, Ik, and Il, stimulated by the soul relation with the family, and of course by the center Icd. The emotions are also classified on the Good/Bad scale, firstly by associating them with primary information and through repetition, stabilized afterwards and consolidated in CASI. The general and rapid development of the body is assured by Icd. During the years of adolescence and youth, the functioning and development of the GTS, translated into consciousness as Ic, brings important changes in the personal behavior mode. The development of GTS is in fact initiated by IGG, and this development is part of the genetic program of evolution of the human being. It also begins to take on a specific importance the center Ia, the image of one's own body outwardly and towards one's own person, playing a special role in making decisions. For the system of relationships with those around, the image of one's own person outwardly is important, and this is modeled not only by own beliefs, but also by the feedback signals from the acquaintances and society in general. In other words, the personality and self-consciousness are outlined, by means of Ia. The Ic center is, from this point of view, an engine for the development and flourishing of one's own personality, and the way in which this is represented in society passes in the forefront. This development also includes the preparation for a professional career, the awareness of the role of training and professional level in connecting to a stable and sufficient source of income for own and for the support of the future family becoming a priority task. Ik is developing therefore as a result of the accumulation of new knowledge at the professional and cultural level. During the maturity years, the centers Ik, Ia, Ic, Iw are working plenary, manifested by the desire to achieve a valuable social status, to obtain important material resources, to gain power and social distinction. It is the time when the body is strong, sustained not only by the center Ia, but also by the connection with $\mathrm{AC}$, the gate of trust and the source of the vital force, which is appealed especially in moments of decline and difficulty.

Ic can be evaluated during this period through productivity, not only in terms of the proliferation and formation of the new generation, but also in achieving the objectives of connection to the necessary resources able to support it. During the old age, marked by the retirement from the institutional activities, as a recognition by society of the weakening of the body vital forces, is characterized by a maximum enrichment of the life experience, after the passage through its main stages, and it is reached the level of wisdom, a source of experience for the others. This evolution is dictated by IGG. While Ik achieves the maximum level of development, the center Ia, a reflection of MIS in consciousness, decreases its functionality level, and Ic reduces its functions, disappearing practically completely. In other words, the mechanisms of infogenetic codification disappear or cease its functions, because the great life project, which is the creation and formation of the next generation, is over. Interpreting these mechanisms in informational terms, we can observe that the "pure" (virtual) information, specific to CASI and CDC systems and manifested by the life experience, is accumulated in memory, attaining a high quantity level, while the proportion of matter-related (codified) information decreases. Regarded from this point of view, it seems that the body is already preparing during this period of detachment of the informational luggage from informed matter, as it was found to occur during the near-death experiences (NDEs).

The associated phenomena were plausibly explained by the information model described above [6]. Moreover, the issue of the immortality [14,15], an "after-life" stage of consciousness, could be discussed in the light of this model, as it has recently been suggested $[16,17]$. On the other hand, as information of a physical body could be 
found on a sphere around it [11], we can extend this statement to the human body, and could conclude that the so controversial bio-field and aura around the human body $[18,19]$, claimed to be observed by the initiated persons of the oriental cultures, could become a favorable issue of discussion, this time from the perspective of such an informational model. From the same model, we can also conclude that it is possible to slow down the ageing process, stimulating by various activities dedicated both to the relation with the body and with the informational system itself, in order to maintain and reinforce the union between the information and informed matter. A "successful aging" could be in general defined by the following main parameters: longevity, health, mental and cognitive efficiency, personal control, social competence and productivity and life satisfaction (wellbeing or destiny) [20]. Each of these parameters deserves therefore a careful attention. Leaving aside the genetic inheritance which each one disposes of, determining a particular longevity, this could be done by continuing to trust in life (Ib), practicing the physic exercises, contributing in this way to the fortification both of the direct communication between CDC (Iw) and the execution elements (EE) and of their power. Specific exercises should be applied to invigorate the activity of the external sensors, in order to maintain a good support and communication with CASI (Ik). Thus, as it was shown recently [21], a minimal but regular program of exercises could contribute to a "successful aging", by the decreasing of the risk of the cardiovascular mortality and osteoporosis, by the prevention of the development of some cancers and by the increasing of the longevity. The life quality and longevity improvement could be synthetically and very suggestively expressed by the following conclusion: "exercise can help add years to life and above all, add life to years" [21]. The quality of the components of the living (informed) matter managed by MIS (Ia) is also important for the health and longevity. The Mediterranean diet is strongly recommended by its abundant composition in legumes, fruits, cereals, vegetables, fish and olive oil, leading to a low risk of mortality from cardiovascular diseases, incidence of the mortality from cancer, and incidence of Parkinson's and Alzheimer's disease [22]. The social life is also an important anti-aging factor, as a natural source, suitable to exercise and improve the efficiency of the sensors, of the memory (CASI (Ik)), of the decisional center (CDC (Iw)) and of the emotional system (IES (Il)), with positive effects on the personal wellbeing and life satisfaction. The connection with the art and the cultural sources enriches also the communication efficiency and the affectivity. A satisfactory social life dedicated to the relation with the family and the society, succeeds to train the mental faculties (memory, attention, reaction capacity) and the intimate affective existence. Moreover, despite of the expected decline of physiological functions, the emotional ageing seems to benefit of the age [23]. This could be probably a consequence of the release from the tasks required by the life great project the growth/formation/education of the descendants - and from the social ones, experienced by Ic.

\section{Conclusions}

We operate between two alternatives, YES and NO, and we have the possibility to choose by our free will one of them. Today's decision influences the trajectory of tomorrow's life and every moment we depend on the choice done in the past. But such a binary operating system is in fact an informational one. According to the last discoveries of quantum mechanics and astrophysics, we can conclude that the universe itself is a bipolar system, which the human being is connected to, operating as reactive dual (infomatter) connected entities, capable and skillful for adaptation by means of a learning process, oriented for the individual survival and the conservation of the species [24]. The human informational system consists of seven interrelated subsystems, but each of them with a distinct functional role, reflected in consciousness by the centers Ik (data library, life experience), Iw (operative center of decision), Il (emotional status), Ia (body current state), Ic (genetic elaboration/transmission), Icd (genetic generator of information inherited from parents) and Ib, which is the gateway to the antientropic component of the universe, with living supporting properties. The life cycle can be analyzed by appealing to the characteristics and the evolution of these centers during the life and to their role for each specific period. Although Icd is not detected by the children during the first years of life through an own awareness process, this center becomes defining for their rapid development and formation. MIS (Ia) accomplishes its basic functions concerning the matter absorption/desorption processes, while Ik, Il and Iw are connected to the family and environment, especially to learn. During the adolescence and then the adulthood period, the informational contribution to the life experience is given especially by the activities of Ic, Ia, Il and Iw centers, in relation with the aspirations related to the family, profession and the position in the society. During the older adult stage, the accumulation of life experience becomes the source of wisdom for the others, and of contemplation of one's own life and its purpose, the center Ib becoming more and more active.

The information baggage increases, while the info-material support decreases, culminating in the detachment of the informational entity from the material one and its passage into an "after-life" stage. However, according to the informational model presented here, it is possible to slow down the ageing process, by the stimulation of various activities dedicated both to the relation with the body and with the informational system itself, in order to maintain and reinforce the union between information and informed matter. This could be done continuing to be interested in life (Ib), following a careful lifestyle, a vegetable-based diet (Ia), practicing the physic exercises (communication between CDC (Iw) and EE), connecting the art and cultural sources, the family and social manifestations, which support the functions of the body, the mental faculties (attention, memory (Ik), reaction capacity (Iw)) and the own affectivity resorts (Il). 


\section{Acknowledgment}

As a continuity of life and information and for further development, the author dedicates this work to his son Adrian Gaiseanu and to his daughter Ana Maria Gaiseanu, followers and contributors to such a healthy and reliable life thinking/driving system. The author gratefully thanks to the members of the Editorial Staff for their support and collaboration.

\section{References}

1. Kaku M (2014) The future of the mind, the scientific quest to understand enhance and empowered the mind, London, UK.

2. Fracasso C, Friedman H (2011) Near-death experiences and the possibility of disembodied consciousness: challenges to prevailing neurobiological and psychosocial theories. Neuro Quantology 9(1): 4153.

3. Gaiseanu F (2017) Quantum-assisted process of disembody under neardeath conditions. Neuro Quantology 15(1): 10-17.

4. Radin D (2006) Entangled minds, extrasensory experiences in a quantum theory, New York, London, Toronto, Sydney: Para view Pocket Books, a division of Simon Shuster, Inc, Newyork, USA.

5. Hameroff S (1998) Quantum computation in brain microtubules? The penrose hameroff. orch or" model of consciousness". philos trans royal society London. Ser A: Math Phys Sci 356(1743): 1869-1898.

6. Gaiseanu F (2017) Consciousness as informational system of the human body. Consciousness and Life Physics. Cosmology and Astrophysics Journal 16(1): 14-25.

7. Gaiseanu F (2017) An Information based model of consciousness fully explaining the mind normal/paranormal properties. Neuro Quantology 15(2): 132-140.

8. Gaiseanu F, Graur A (2018) Cognitive centers related attitude: Application for an iterative evaluation method in music-based therapy process, Abstract book of the conference on science of consciousness (Co- Chair Prof. Stuart Hameroff, The University of Arizona-Center for Consciousness Studies, Tucson, Arizona), Consciousness and Education - Cognitive Development, Concurrent Session C27, p. 165.

9. Hajdukovic D (2010) Dark matter, dark energy and gravitational properties of antimatter.
10. Hajdukovic D (2013) Can observations inside the solar system reveal the gravitational properties of the quantum vacuum. Astrophys Space Sci 343: 505-509.

11. Verlinde E (2010) On the origin of gravity and the laws of newton, institute for theoretical physics university of amsterdam valckenierstraat 651018 XE, Amsterdam, Netherlands.

12. Carroll S (2015) The arrow of time and from eternity to here, the quest for the ultimate theory of time, GB: One world publication.

13. Gaiseanu F (2018) Information from philosophic to physics concepts for informational modeling of consciousness, Philosophy Study 8(8): 368382.

14. Dispenza D (2007) Evolve your brain: The science of changing your mind, Deerfield beach, Health communications, Inc, Florida, USA.

15. Hameroff S, Chopra D $(2010,2017)$ Can science explain the soul?

16. Meijer DKF (2013) Immortality: Myth or becoming reality? On the conservation of information. Syntropy 2013(3): 166-203.

17. Gaiseanu F (2018) Near-death experiences and immortality from the perspective of an informational modeling of consciousness. Gerontology \& Geriatrics Studies 2(3): 1-3

18. Meijer DKF (2013) Information: what do you mean? On the formative element of our universe. Syntropy 3: 1-49.

19. Stenger JV (1999) Bioenergetic fields, The scientific review of alternative medicine. Spring/Summer 3(1): 1

20. Kafatos CM, Chevalier G, Chopra D, Hubacher J, Kak S, et al. (2015) Biofield science: current physics perspectives. Glob Adv Health Med 4(Suppl): 25-34.

21. Baltes P, Baltes M (1990) Psychological perspectives on successful aging: The model of selective optimization with compensation. In: Baltes $\mathrm{P}$, Baltes M (Eds.), Successful Aging, pp. 1-34.

22. Gremeaux V, Gayda M, Lepers R, Sosner P, Juneau M, et al. (2012) Exercise and longevity. Maturitas 73(4): 312-317

23. Sofi F, Cesari F, Abbate R, Gensini GF, Casini A, et al. (2008) Adherence to mediterranean diet and health status: meta-analysis. BMJ 337(2): a1344.

24. Scheibe S and Carstensen L (2010) Emotional aging: recent findings and future trends. Journal of Gerontology: Psychological Sciences 65B(2): 135-144.
Creative Commons Attribution 4.0

International License

For possible submissions Click Here

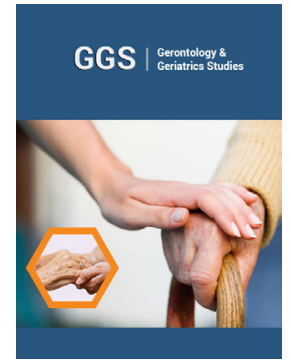

Gerontology \& Geriatrics Studies

\section{Benefits of Publishing with us}

- High-level peer review and editorial services

- Freely accessible online immediately upon publication

- Authors retain the copyright to their work

- Licensing it under a Creative Commons license

- Visibility through different online platforms 